Document downloaded from:

http://hdl.handle.net/10251/35357

This paper must be cited as:

Hernández Orallo, E.; Serrat Olmos, MD.; Cano Escribá, JC.; Tavares De Araujo Cesariny Calafate, CM.; Manzoni, P. (2012). Improving selfish node detection in MANETs using a collaborative watchdog. IEEE Communications Letters. 16(5):642-645. doi:10.1109/LCOMM.2012.030912.112482.

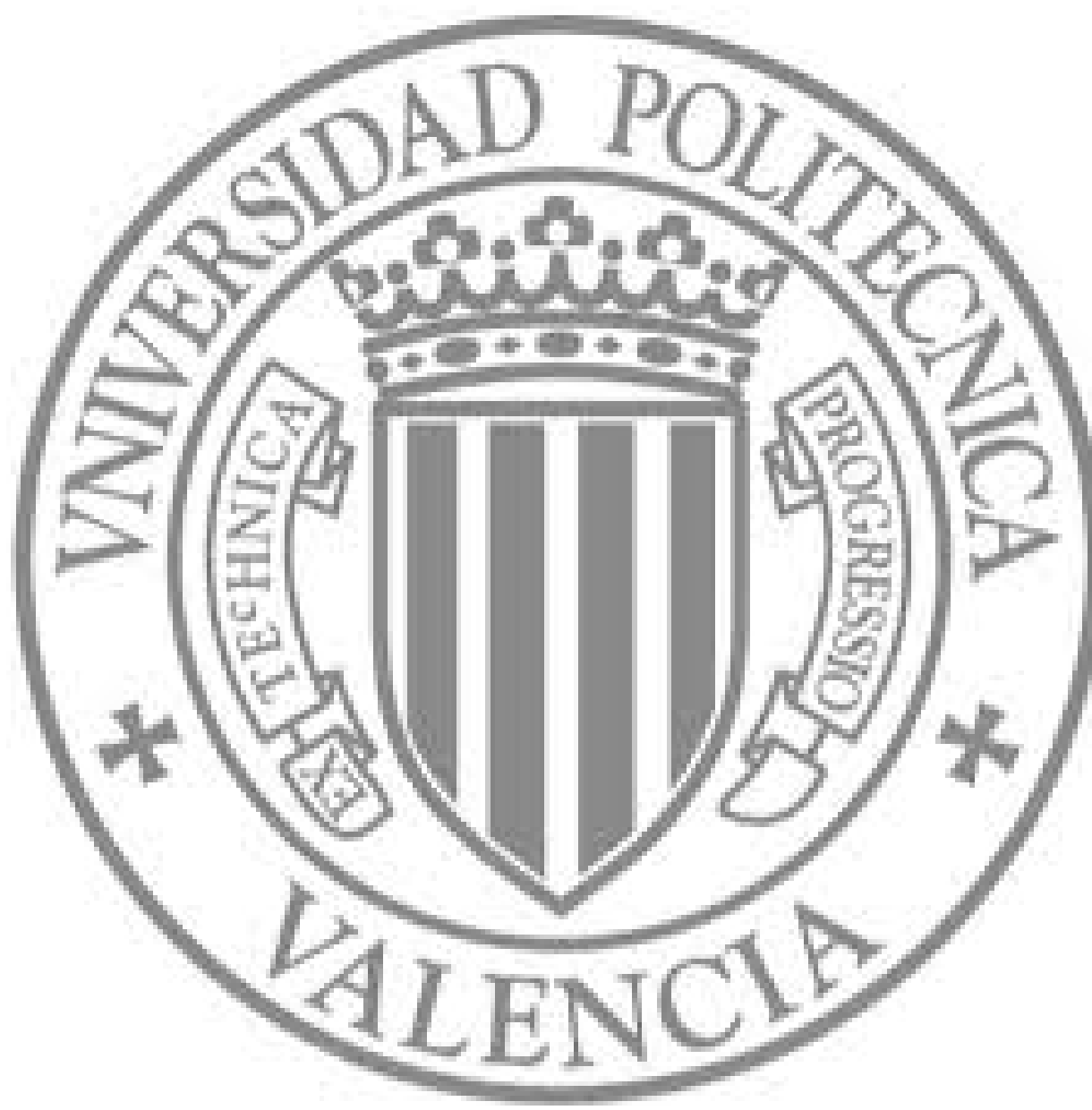

The final publication is available at

http://dx.doi.org/10.1109/LCOMM.2012.030912.112482

Copyright Institute of Electrical and Electronics Engineers (IEEE) 


\title{
Improving Selfish Node Detection in MANETs Using a Collaborative Watchdog
}

\author{
Enrique Hernández-Orallo, Manuel D. Serrat, Juan-Carlos Cano, Carlos T. Calafate, Pietro Manzoni
}

\begin{abstract}
Mobile ad-hoc networks (MANETs) are composed of mobile nodes connected by wireless links without using any pre-existent infrastructure. MANET nodes rely on network cooperation schemes to properly work, forwarding traffic unrelated to its own use. However, in the real world, most nodes may have a selfish behavior, being unwilling to forward packets for others in order to save resources. Therefore, detecting these nodes is essential for network performance.

Watchdogs are used to detect selfish nodes in computer networks. A way to reduce the detection time and to improve the accuracy of watchdogs is the collaborative approach. This paper proposes a collaborative watchdog based on contact dissemination of the detected selfish nodes. Then, we introduce an analytical model to evaluate the detection time and the cost of this collaborative approach. Numerical results show that our collaborative watchdog can dramatically reduce the overall detection time with a reduced overhead.
\end{abstract}

Index Terms-MANET, Selfish nodes, Performance Evaluation

\section{INTRODUCTION}

MANETs are used in various contexts like intelligent transportation systems, mobile social networks, emergency deployment, etc. In a MANET, nodes can freely move around while communicating with each other. These networks may underperform in the presence of nodes with a selfish behaviour, particularly when operating under energy constraints. A selfish node will typically not cooperate in the transmission of packets, seriously affecting network performance. Although less frequent, nodes may also fail to cooperate either intentionally (a malicious behaviour) or due to faulty software or hardware.

We consider that watchdogs are the appropriate mechanism to detect these situations [1]. Essentially, watchdog systems overhear wireless traffic and analyse it to decide if neighbouring nodes are not cooperating. Several works have studied the impact of node selfishness on MANETs proposing different detection mechanisms [2]-[8]. In [1], a bayesian watchdog was introduced, as a way to improve the accuracy of the detection.

\section{A Collaborative Watchdog}

A way to reduce the detection time of selfish (or noncooperative) nodes in a network is the collaborative watchdog. Although some of the aforementioned papers ( [2], [4], [8]) introduced some degree of collaboration on their watchdog schemes, the diffusion was very costly (usually based on sending periodic messages).

This paper introduces an efficient approach to reduce the detection time of selfish nodes based on contact dissemination.

The authors are with the Departamento de Informática de Sistemas $y$ Computadores. Universitat Politècnica de València. Spain. (contact email: ehernandez@disca.upv.es)
If one node has previously detected a selfish node using its watchdog it can spread this information to other nodes when a contact occurs. We say that a node has a positive if it knows the selfish node. The detection of contacts between nodes is straightforward using the node's watchdog. Notice that the watchdog is overhearing the packets of the neighbourhood; thus, when it starts receiving packets from a new node it is assumed to be a new contact. Then, the node transmits one message including all known positives it knows to this new contacted node. The number of messages needed for this task is the overhead of the collaborative watchdog.

Formally, we have a network of $N$ wireless mobile nodes, with $C$ collaborative nodes and $S$ selfish nodes. Initially, the collaborative nodes have no information about the selfish nodes. A collaborative node can have a positive when a contact occurs in the following way:

- Selfish contact: one of the nodes is the selfish node. Then, the collaborative node can detect it using its watchdog and have a positive about this selfish node. Nevertheless, a contact does not always imply a detection. To model this fact, we introduce a probability of detection $\left(p_{d}\right)$. This probability depends on the effectiveness of the watchdog and the type of contact (for example if the contact time is very low, the watchdog does not have enough information to evaluate if the node is selfish or not).

- Collaborative contact: both nodes are collaborative. Then, if one of them has one or more positives, it can transmit this information to the other node; so, from that moment, both nodes have these positives. As in the selfish contact case, a contact does not always imply a collaboration. We model this with the probability of collaboration $\left(p_{c}\right)$. The degree of collaboration is a global parameter of the network to be evaluated. This value is used to reflect that either a message with the information about the selfish nodes is lost or that a node temporally does not collaborate (for example, due to a failure or simply because it is switched off). In real networks, full collaboration $\left(p_{c}=1\right)$ is almost impossible.

Although defining a reaction scheme is out of the scope of this paper, there are basically two approaches in the literature: isolation and incentivation. Isolation methods are intended to keep the misbehaving nodes outside the network, excluding them from all kinds of communication. Incentivation methods try to convince the selfish nodes to change their behaviour, and become collaborative instead of selfish, using a virtual payment scheme or a similar mechanism.

\section{Performance Model}

The goal of this section is to obtain a model to evaluate the time and cost of detecting selfish nodes on a network 
with collaborative watchdogs. The network is modeled as a set of $N$ wireless mobile nodes, with $C$ collaborative nodes and $S$ selfish nodes $(N=C+S)$. It is assumed that the occurrence of contacts between two nodes follows a Poisson distribution $\lambda$. This assumption has been shown to hold in several mobility scenarios of both human and vehicles [9][12]. For example, in [9] a useful expression is derived for obtaining $\lambda$ from the parameters of the random waypoint and random direction models.

First, we derive a basic model for $S=1$. In this case, a collaborative node has 2 states: NOINFO, when the node has no information about the selfish node, and POSITIVE when the node knows who the selfish node is (it has a positive). All nodes have an initial state of NOINFO and they can change their initial state when a contact occurs. Using a contact rate $\lambda$ we can model the network using a Continuous Time Markov Chain (CTMC) with states $s_{i}=(c)$, where $c$ represents the number of collaborative nodes in the POSITIVE state. At the beginning, all nodes are in NOINFO state. Then, when a contact occurs, $c$ can increase by one. The final (absorbing) state is when $c=C$. So, this can be modelled using a CTMC with an initial state $s_{1}=(0), \tau=C$ transient states, and one $(v=1)$ absorbing state $s_{\tau+1}=(C+1)$. Then, the transition matrix $P$ in canonical form is:

$$
\mathbf{P}=\left(\begin{array}{cc}
\mathbf{Q} & \mathbf{R} \\
\mathbf{0} & \mathbf{I}
\end{array}\right)
$$

where $\mathbf{I}$ is a $v \times v$ identity matrix (in this case 1 ), $\mathbf{0}$ is a $v \times \tau$ zero matrix, $\mathbf{Q}$ is a $\tau \times \tau$ matrix with elements $p_{i j}$ denoting the transition rate from transient state $s_{i}$ to transient state $s_{j}$ and $\mathbf{R}$ is a $\tau \times v$ matrix with elements $p_{i j}$ denoting the transition rate from transient state $s_{i}$ to the absorbing state $s_{j}$.

Now, we derive the transition rates $p_{i j}$. Given a state $s_{i}=$ (c) the following transitions can occur:

- $(c)$ to $(c+1)$ : This case takes place when a collaborative node changes from NOINFO state to POSITIVE state. The transition probability is $t_{c}=\left(\lambda p_{d}+\lambda p_{c} c\right)(C-c)$. The term $\lambda p_{d}$ represents the probability of detection of a selfish node (using the watchdog) and $\lambda p_{c} c$ the probability of transmission for the information of the selfish node (it depends on $c$, so this probability is greater if more nodes are in the POSITIVE state). Finally, factor $(C-c)$ represents the number of pending nodes.

- $(c)$ to $(c)$ : This is the probability of no changes, and its value is $t_{0}=1-t_{c}$.

Using the transition matrix $P$ we can derive two different expressions: one for the detection time $T_{d}$ and another one for the overall overhead (or cost) $M_{d}$. We start with the detection time. Using the fundamental matrix $\mathbf{N}=(\mathbf{I}-\mathbf{Q})^{-1}$, we can obtain a vector $\mathbf{t}$ of the expected time to absorption as $\mathbf{t}=\mathbf{N v}$, where $\mathbf{v}$ is a column vector of ones $\left(\mathbf{v}=[1,1, \ldots, 1]^{T}\right)$. Each entry $t_{i}$ of $\mathbf{t}$ represents the expected time to absorption from state $s_{i}$. Since we only need the expected time from state $s_{1}=(0)$ to absorption, the detection time $T_{d}$, is:

$$
T_{d}=\mathbf{v}_{\mathbf{1}} \mathbf{N} \mathbf{v}
$$

where $\mathbf{v}_{\mathbf{1}}=[1,0, \ldots, 0]$.
For obtaining the overall overhead (or transmission cost) we need to obtain the number of transmitted messages for each state $s_{i}$. During state $s_{1}$ no node is in the POSITIVE state. In this state, no messages are transmitted and $m_{1}=0$. The second state $s_{2}$ starts when 1 node has a POSITIVE state (that is, there is one sender). In this case, this POSITIVE can be transmitted to all nodes (except itself) for the duration of this state (denoted as $f_{2}$ ) with a rate $\lambda$ and probability $p_{c}$. Then, the expected number of messages can be obtained as $m_{2}=f_{2} \lambda(C-1) p_{c}$. For state $s_{3}$, we have 2 possible senders, so $m_{3}=2 f_{3} \lambda(C-1) p_{c}$. Then, for state $s_{i}$ we have $(i-1)$ senders, so $m_{i}=(i-1) f_{i} \lambda(C-1) p_{c}$. We can obtain the duration of each state using the fundamental matrix $\mathbf{N}$. By definition, the elements of the first row of $\mathbf{N}$ are the expected times in each state starting from state 0 . Then, the duration of state $s_{i}$ is $f_{i}=\mathbf{N}(1, i)$. Summing up, the cost of transmission is:

$$
M_{d}=\lambda(C-1) p_{c} \sum_{i=1}^{\tau} \Phi\left(s_{i}\right) \mathbf{N}(1, i)
$$

where $\Phi\left(s_{i}\right)=(i-1)$ is the number of senders in state $s_{i}$.

We can now extend the previous basic model to the case of several selfish nodes $(S>1)$. The solution is based on using a Continuous Time Markov Chain with $\mathrm{S}$ dimensions. We start with $S=2$, so we have a two-dimensions CTMC (for short, a 2D-CTMC). Each state $s_{i}$ now has two values $\left(c_{2}, c_{1}\right)$, where $c_{1}$ is the number of collaborative nodes having a POSITIVE for selfish node 1 , and $c_{2}$ is the same for selfish node 2 . At the beginning all nodes are in the NOINFO state. Then, when a contact occurs, $c_{1}$ and $c_{2}$ can increase by one. The final (absorbing) state is when $\left(c_{2}, c_{1}\right)=(C, C)$. So, the 2D-CTMC has an initial state $s_{1}=(0,0), s_{\tau}=(C+1)^{2}-1$ transient states (from $s_{1}=(0,0)$ to $s_{\tau}=(C-1, C)$ state) and $v=1$ absorbing state $s_{\tau+1}=(C, C)$. Now, we derive the transition rates $p_{i j}$ for the transition matrix. Given the state $s_{i}=\left(c_{2}, c_{1}\right)$, the following transitions can occur:

- $\left(c_{2}, c_{1}\right)$ to $\left(c_{2}, c_{1}+1\right)$ : the same that in $S=1$ model, replacing $c$ by $c_{1}, t_{c 1}=\left(\lambda p_{d}+\lambda p_{c} c_{1}\right)\left(C-c_{1}\right)$

- $\left(c_{2}, c_{1}\right)$ to $\left(c_{2}+1, c_{1}\right)$ : the same for $c_{2}, t_{c 2}=\left(\lambda p_{d}+\right.$ $\left.\lambda p_{c} c_{2}\right)\left(C-c_{2}\right)$

- $(c, c)$ to $(c, c): t_{0}=1-t_{c 1}-t_{c 2}$

and, using equation 2 , we can obtain the detection time $\left(T_{d}\right)$. We can extend this model to the case of $S>2$. Then we have $\tau=(C+1)^{S}-1$ transient states and, for each state $s_{i}=\left(c_{S}, c_{S-1}, \ldots c_{2}, c_{1}\right)$, the transition rate from $c_{j}$ to $c_{j}+1$ is $t_{c j}=\left(\lambda p_{d}+\lambda p_{c} c_{j}\right)\left(C-c_{j}\right)$.

For the overhead, we assume that a node transmits only one message for all the positives it has. Then, the number of messages in each state depends on the distribution of the positives. Obtaining all the combinations when $S$ is high can be very complex, but a simple approximation based on bounding the value of senders can be used. It is easy to see that the number of senders in each state is between the maximum of $c_{j}$ and the minimum between the sum of $c_{j}$ and $C$. That is, $\max \left(s_{i}\right) \leq \Phi\left(s_{i}\right) \leq \min \left(\operatorname{sum}\left(s_{i}\right), C\right)$ where $\max \left(s_{i}\right)=\max _{j=1}^{S} c_{j}$ and $\operatorname{sum}\left(s_{i}\right)=\sum_{j=1}^{S} c_{j}$ Then, we estimate the number of senders $\Phi\left(s_{i}\right)$ by calculating the 
mean of the lower and upper bounds. Finally, the number of messages is obtained using equation 3 .

Now, we briefly describe the validation process of the model previously presented. This performance model obtains the time and overhead $\left(T_{d}, M_{d}\right)$ from the following set of inputs: the rate of contacts $(\lambda)$, the network $(N, C, S)$ and the watchdog $\left(p_{c}, p_{d}\right)$ parameters. We used the ns-2 setdest command to create contact traces, which are used, on the one hand to fit the $\lambda$ value that is used in our performance model and on the other hand to simulate the contacts to obtain the simulation results. We validate our model using a set of 100 random tests. The tests have different parameters values $\left(N, C, S, p_{c}, p_{d}\right)$ and mobility patterns (mean speed of nodes $v$, communication range $r$, side $l$, etc.). For each test, we repeated the simulation 1000 times in order to obtain values with confidence intervals for the detection time and the overhead. These values are compared with the results of our model in order to obtain the accuracy of our model. After running all the tests we obtained the mean error (and 95\% confidence intervals) for $T_{d}$ and $M_{d}$. For the detection time the mean relative error was $2.18 \%([0.52,3.95])$ and for the overhead it was $2.86 \%([0.77$, $6.48])$. These results confirms that the error of our model is very low.

\section{EVALUATION RESULTS}

This section is first devoted to evaluating the performance of our collaborative watchdog using the performance model detailed in section III. All the model were implement and evaluated using Matlab. For the following evaluations we consider a contact rate of 0.0135 contacts $/ \mathrm{h}, \lambda_{v}=3.71 \times 10^{-6} \mathrm{~s}^{-1}$. This value was calculated in [12] based on real motion traces from about 2100 operational taxis for about one month in Shanghai city.

The first evaluation shows the influence of the degree of collaboration in a network with 50 nodes and one selfish node (see figure 1a) with different detection probabilities values $\left(p_{d}\right)$. We can see that increasing the degree of collaboration from 0 to 0.2 reduces the detection time exponentially and increases the overhead (cost) exponentially as well. This reduction is quite significant for low detection probabilities $\left(p_{d}=0.1\right)$. For $p_{c}=0$ (no collaboration), the detection time is $12 \times 10^{6} \mathrm{~s}$ (about 3300 hours). This value can be greatly reduced by using our collaborative watchdog. Thus, if all nodes implement the collaborative approach $\left(p_{c}=1\right)$ the detection time is reduced to 30 hours. Even for a low collaboration rate $\left(p_{c}=0.2\right)$ the time is reduced to 78 hours. For both cases, the overhead is approximately of 210 messages (less than 7 messages by hour, a very reduced cost). We can also see that increasing the probability of collaboration (from 0.4 to 1 ) has low impact on both the detection time and the overhead, which emphasizes on the resilience of our collaborative approach.

The second evaluation shows the impact of the number of nodes ranging from 0 to 100 (see figure $1 \mathrm{~b}$ ). Three different sets of values for $p_{c}$ and $p_{d}$ were used. The first set $(1,0.8)$ is a full collaborative network with a high probability of detection, the second set has a reduced degree of collaboration $(0.7)$, and

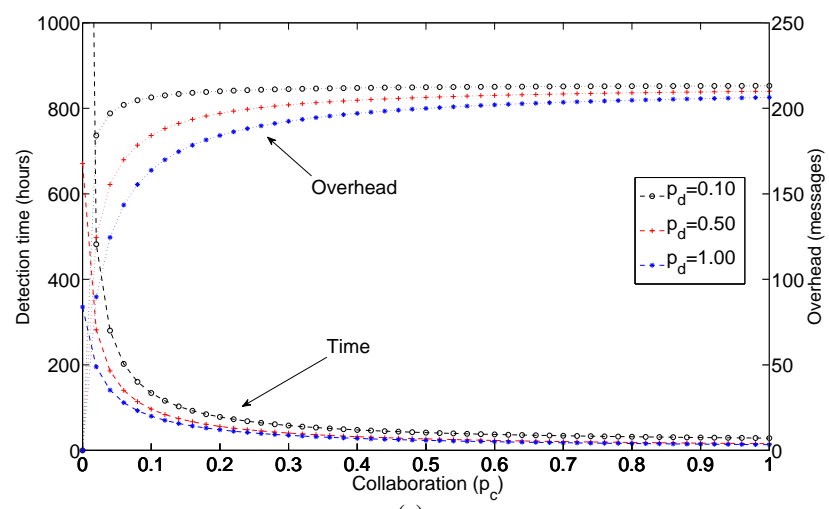

(a)

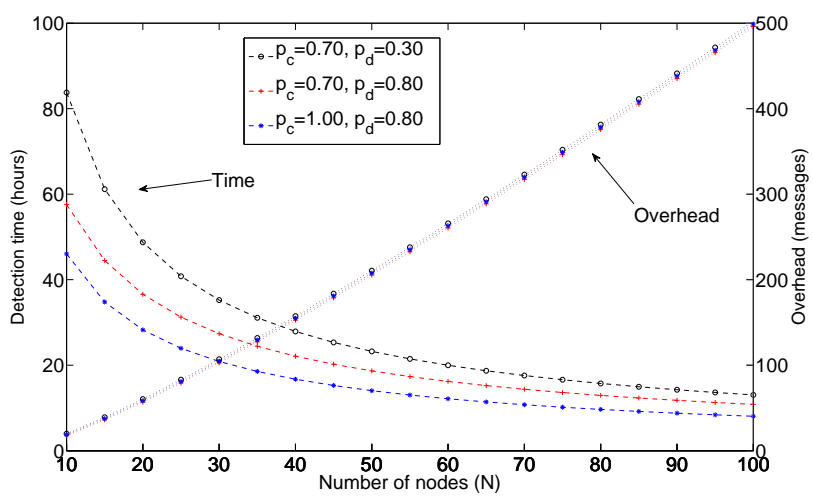

(b)

Fig. 1: Evaluation for $S=1$. a) depending on $p_{c}$ b) depending on $\mathrm{N}$.

finally the last set has a low probability of detection (0.3). We observe that, in general, the greater the number of nodes, the lesser the detection time and the greater the number of messages. As expected, reduced values of collaboration and detection probabilities imply greater detection times.

Figure 2 shows the influence of the number of selfish nodes $S$ for $N=50$. As expected, the detection time increases when the number of selfish nodes is higher. Regarding the overhead, we can see that the number of messages increases exponentially for low values of $S$, and then it decreases slowly, for $S>10$. The reason is that, when the number of selfish nodes is high, the collaborative nodes are reduced and they can transmit fewer messages.

More experiments were performed using different $\lambda$ values, for example with a contact rate of 0.101 contacts $/ \mathrm{h}$, obtained from human mobility traces [7], and the results obtained were similar to those presented here.

Now we proceed to compare our collaborative watchdog approach with previous cooperative approaches that use periodic messages for the diffusion of information about positives detections. If a node has information about a positive, it will periodically broadcast a message with a given period $P$. This message will be received by all nodes that are within the communication range of the sender. The performance of this protocol clearly depends on the period $P$. A short period will reduce the detection time, but the number of messages transmitted (the overhead) will be high. A large period will increase the detection time by reducing the overhead. The comparison of both protocols was based on simulations. We 


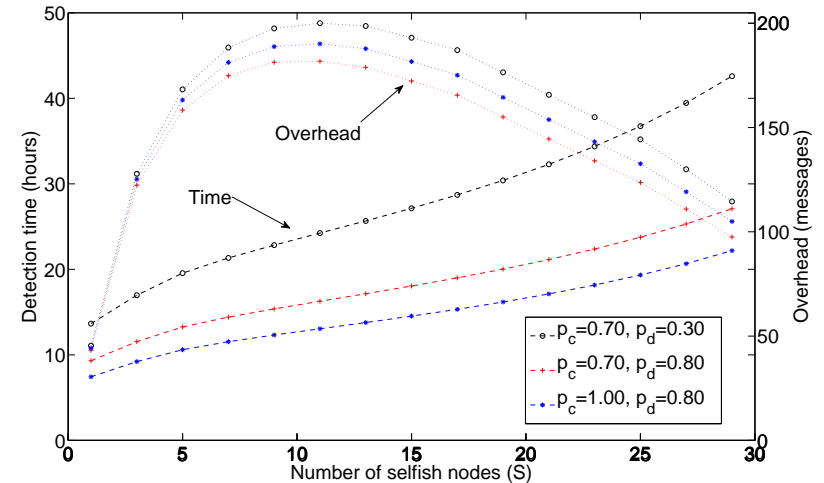

Fig. 2: Evaluation depending on $S$ for $N=50$

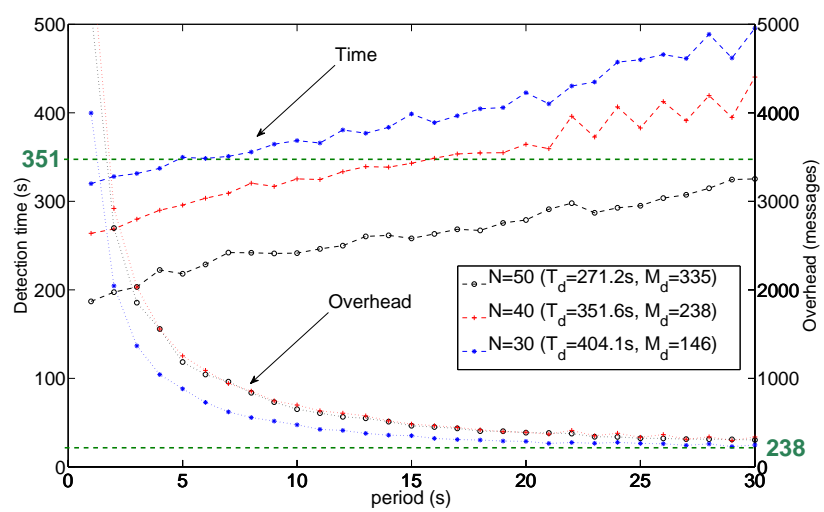

Fig. 3: Detection time and overhead depending on period $P$ for the periodic approach. The main parameters for the mobility model are mean-speed $=$ $5 \mathrm{~m} / \mathrm{s}$, side-area $=1000 \mathrm{~m}$, pause-interval $=1 \mathrm{~s}$, range $=100 \mathrm{~m}$

implemented the periodic diffusion protocol, as described in the previous paragraph. By using the ns-2 setdest command we generate mobility scenarios that are used to simulate both approaches.

Figure 3 shows the detection time and overhead for $P$ ranging from 1 to $30 \mathrm{~s}$ for the periodic diffusion protocol with three different number of nodes. The results confirms that increasing the period $P$ implies that the detection time is increased and the overhead reduced. We can compare these results with the detection time and overhead values for our collaborative watchdog (that are in the legend of the plot). For example, for $N=40$, the periodic diffusion for periods below $15 \mathrm{~s}$ has a shorter detection time than our model but with a higher overhead. For example, for $P=5 \mathrm{~s}$, the detection time is 295 s (a reduction of $15 \%$ ) and the overhead is 1253 messages (an increment of 526\%). For $P=15 \mathrm{~s}$, the detection time is similar to our approach, and the overhead is 483 messages (205\% higher). We conclude that, although using periodic diffusion can reduce the detection time slightly, this implies a large overhead.

\section{CONCLUSIONS}

In this paper we have proposed and evaluated a new collaborative watchdog approach. We modelled its performance using a Continuous Time Markov Chain with two parameters to indicate the degree of collaboration and detection of the watchdog. Numerical results show that a collaborative watchdog can reduce the overall detection time with a reduced cost in term of message overhead. This reduction is very significant when the watchdog detection effectiveness is low. Furthermore, this reduction can be obtained even with a moderate degree of collaboration.

As future work, we plan to extend this model to evaluate the effect of false positives and false negatives. Such extension poses several problems: first, a node needs to transmit not only the positives but also the negatives, so it will increase the overhead; second, when a node receives this information about positives and negatives, conflicts with previous information may appear (for example, when a node has a negative about a given node and it receives a positive). So, an updating strategy may be needed. We also plan to evaluate the case of malicious or cheating behavior by introducing some kind of reputation scheme. Finally, we are also planning to implement this collaborative watchdog in a testbed.

\section{ACKNOWLEDGMENTS}

This work was partially supported by the Ministerio de Ciencia e Innovación, Spain (Grant TIN2011-27543-C03-01).

\section{REFERENCES}

[1] J. Hortelano, J. C. Ruiz, and P. Manzoni, "Evaluating the uselfusness of watchdogs for intrusion detection in vanets," in In ICC'10 Workshop on Vehicular Networking and Applications, 2010.

[2] S. Marti, T. J. Giuli, K. Lai, and M. Baker, "Mitigating routing misbehavior in mobile ad hoc networks," in Proceedings of MobiCom, 2000, pp. 255-265.

[3] K. Paul and D. Westhoff, "Context aware detection of selfish nodes in dsr based ad-hoc networks," in In Proceedings of IEEE Globecom, 2002.

[4] S. Buchegger and J.-Y. Le Boudee, "Self-policing mobile ad hoc networks by reputation systems," Communications Magazine, IEEE, vol. 43, no. 7, pp. 101-107, jul 2005.

[5] M. Karaliopoulos, "Assessing the vulnerability of dtn data relaying schemes to node selfishness," Communications Letters, IEEE, vol. 13, no. 12, pp. 923-925, dec 2009.

[6] Y. Li, P. Hui, D. Jin, L. Su, and L. Zeng, "Evaluating the impact of social selfishness on the epidemic routing in delay tolerant networks," Communications Letters, IEEE, vol. 14, no. 11, pp. 1026-1028, nov 2010.

[7] Y. Li, G. Su, D. Wu, D. Jin, L. Su, and L. Zeng, "The impact of node selfishness on multicasting in delay tolerant networks," Vehicular Technology, IEEE Trans. on, vol. 60, no. 5, pp. 2224-2238, jun 2011.

[8] H. Otrok, M. Debbabi, C. Assi, and P. Bhattacharya, "A cooperative approach for analyzing intrusions in mobile ad hoc networks," in Distributed Computing Systems Workshops, 2007. ICDCSW '07. 27th International Conference on, june 2007, p. 86.

[9] R. Groenevelt, P. Nain, and G. Koole, "The message delay in mobile ad hoc networks," Performance Evaluation, vol. 62, pp. 210-228, Oct 2005.

[10] T. Karagiannis, J.-Y. Le Boudec, and M. Vojnović, "Power law and exponential decay of inter contact times between mobile devices," in Proceedings of MobiCom, New York, NY, USA, 2007, pp. 183-194.

[11] W. Gao, Q. Li, B. Zhao, and G. Cao, "Multicasting in delay tolerant networks: a social network perspective," in Proceedings of MobiHoc, New York, NY, USA, 2009, pp. 299-308.

[12] H. Zhu, L. Fu, G. Xue, Y. Zhu, M. Li, and L. M. Ni, "Recognizing exponential inter-contact time in vanets," in Proceedings of INFOCOM. Piscataway, NJ, USA: IEEE Press, 2010, pp. 101-105. 